\title{
A Variational Approach to a Nonlocal Elliptic Problem with Sign-Changing Nonlinearity
}

\author{
Francisco Julio S.A. Corrêa * \\ Universidade Federal de Campina Grande \\ Centro de Ciências e Tecnologia \\ Unidade Acadêmica de Matemática e Estatística \\ CEP:58.109-970, Campina Grande - PB - Brazil \\ E-mail: fjsacorrea@gmail.com \& julio@dme.ufcg.edu.br \\ $\&$ \\ Manuel Delgado \& Antonio Suárez \\ Universidad de Sevilla \\ Dpto. Ecuaciones Diferenciales y Análisis Numérico \\ Sevilla-Spain \\ E-mail: suarez@us.es \& madelgado@us.es
}

\begin{abstract}
In this paper we are concerned with the nonlocal elliptic problem

$$
\left\{\begin{aligned}
-\Delta u & =f(u)\left[\int_{\Omega} g(u)\right]^{p} & \text { in } \quad \Omega \\
u & =0 & \text { on } \quad \partial \Omega
\end{aligned}\right.
$$

where $\Omega \subset \mathbb{R}^{N}$ is a bounded smooth domain, $f, g: \mathbb{R} \rightarrow \mathbb{R}$ are given functions and $p$ is a fixed real number. We use variational methods to prove multiplicity results.
\end{abstract}

*Partially supported by CNPq-Brazil Proc. 301603/2007-3 


\section{Introduction}

In this paper we deal with a class of elliptic nonlocal problems whose prototype is

$$
\left\{\begin{aligned}
-\Delta u & =f(u)\left[\int_{\Omega} g(u)\right]^{p} & \text { in } \quad \Omega, \\
u & =0 & \text { on } \partial \Omega,
\end{aligned}\right.
$$

where $\Omega \subset \mathbb{R}^{N}, N \geq 1$, is a bounded smooth domain, $f, g: \mathbb{R} \rightarrow \mathbb{R}$ are given functions and $p$ is a fixed real number.

Nonlocal problems have been intensively studied since their first appearance in the work of Kirchhoff [24] who studied a wave equation which is a generalization of the D' Alembert equation. On this subject the reader may also consult Carrier [8] and Lions [28].

However, non-local problems are not restricted to mechanical motivations as in the aforementioned works. They also appears in a wide variety of applications as population dynamics (Chipot [14], [15], [13], [16], [17], [18]), Ohmic heating (Freitas [21] and Lacey [27]), the formation of shear bands in materials (Olmstead [30] and Bebernes [4]), heat transfer in thermistors ( Fowler [20]), combustion theory (Pao [31]), the electric ballast resistor (Chafee [11]), microwave heating of ceramic materials (Bose-Kriegsmann [5] and Kriegsmann [26]).

In particular, the present work was motivated by Gomes-Sanchez [22] who studied a variational counterpart of problem (1.1), namely

$$
\left\{\begin{aligned}
-\Delta u & =f(u)\left[\int_{\Omega} F(u)\right]^{p} & \text { in } \quad \Omega, \\
u & =0 & \text { on } \partial \Omega,
\end{aligned}\right.
$$

where $f: \mathbb{R} \rightarrow \mathbb{R}$ is a given function, with a sort of exponential growth, and $F(t)=\int_{0}^{t} f(s) d s$

Problems like (1.2),

$$
\left\{\begin{aligned}
-\Delta u & =\delta \frac{e^{u}}{\int_{\Omega} e^{u}} & \text { in } & \Omega \\
u & =0 & \text { on } & \partial \Omega
\end{aligned}\right.
$$


and

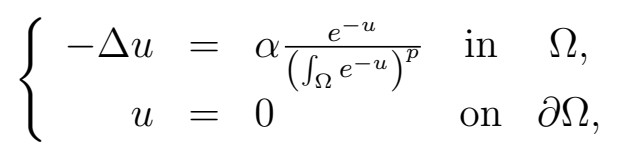

were previously studied by several authors as Bebernes-Lacey [3] and Caglioti-Lions-Marchiori-Pulvirenti [7] and appear in problems related with the theory of gravitational equilibrium of polytropic stars, the fully turbulent behavior of a real flow, among others.

Here, we attack cases in which $f$ changes sign and the area of the bumps of the graph of $f$ plays a key role. Multiplicity of solutions are obtained by exploring these geometric properties. At least to our knowledge, these types of nonlinearities have not been previously studied in the context of nonlocal problems.

In Section 2 we study the case in which $f(0) \geq 0$ and $f$ is positive near zero.

In Section 3 we attack the problem in which $f(0)=0$ and $f$ is negative near zero.

In Section 4 we approach the semipositone case.

Acknowledgement. This work was done while the first author was visiting Dpto. de Ecuaciones Diferenciales y Análisis Numérico - Universidad de Sevilla - Spain in a Post-Doctoral program and was supported by Universidade Federal de Campina Grande and CNPq - Brazil (Proc. 201214/2008-3). Also, the first author thanks M. Delgado, A. Suarez and the staff of the aforementioned Department for their warm hospitality.

\section{$2 \quad f(0) \geq 0$ and $f>0$ near 0}

Here we will study problem (1.2) by establishing a multiplicity result related with the number of zeroes of $f$ and the areas of the bumps of the graph of $f$. More precisely, we will suppose that $f:[0,+\infty) \rightarrow \mathbb{R}$ is a continuous 
function of class $C^{1}$ in $(0,+\infty)$ and satisfies

$$
\liminf _{t \rightarrow 0^{+}} \frac{f(t)}{t^{\alpha}}>0
$$

where $\alpha, p$ are positive numbers satisfying

$(\alpha p)_{1}$

$$
1>\alpha+\alpha p+p
$$

$\left(f_{2}\right) \quad\left\{\begin{array}{c}\text { There are numbers } 0<\theta_{1}<\theta_{2}<\theta_{3} \text { such that } \\ f\left(\theta_{1}\right)=f\left(\theta_{2}\right)=f\left(\theta_{3}\right)=0, f(t)>0 \text { if } 0<t<\theta_{1} \\ f(t)<0 \text { if } \theta_{1}<t<\theta_{2} \text { and } f(t)>0 \text { if } \theta_{2}<t<\theta_{3}\end{array}\right.$

$$
\begin{gathered}
F\left(\theta_{2}\right)=\int_{0}^{\theta_{2}} f(s) d s>0 \\
F\left(\theta_{3}\right)>F\left(\theta_{1}\right) .
\end{gathered}
$$

We point out that condition $\left(F_{1}\right)$, in particular, implies that $F(t)>0$ for all $0<t<\theta_{3}$ (in this way the term $\left[\int_{\Omega} F(u)\right]^{p}$ makes sense), while condition $\left(F_{2}\right)$ tells us that the area of the bump of the graph of $f$ between $\left(\theta_{1}, \theta_{2}\right)$ is less than the area of the bump of the graph of $f$ between $\left(\theta_{2}, \theta_{3}\right)$.

We will suppose that $\Omega$ contains and it is near, in a sense that will be clarified later, a ball $B_{R}=\left\{x \in \mathbb{R}^{N} ;|x|<R\right\}$ with $R$ sufficiently large such that $R^{(N-1) p}>1, N \geq 2$. This is equivalent to

$$
R^{(N-1)(p+1)}>R^{N-1}
$$

and will be strongly used in the proof of the second solution.

Note that the assumption $(\alpha p)_{1}$ implies that $0<\alpha<1$ and assuming $\left(f_{1}\right)$ and if $f(0)=0$, then $f$ is not differentiable in 0 . Indeed, $f_{+}^{\prime}(0)=+\infty$ and so $\left(f_{1}\right)$ is a kind of sublinearity at 0 . 
For this fixed $\Omega$ we choose $\epsilon>0$ sufficiently small such that $(\alpha p)_{2}$

$$
\frac{\left[\int_{\Omega} \varphi_{1}^{\alpha+1}\right]^{p+1}}{\int_{\Omega} \varphi_{1}^{2}} \geq \epsilon^{2-(\alpha+1)(p+1)},
$$

where $\varphi_{1}>0$ is an eigenfunction of $\left(-\Delta, H_{0}^{1}(\Omega)\right)$, associated to the first

eigenvalue $\lambda_{1}$. This is possible because $\frac{\left[\int_{\Omega} \varphi_{1}^{\alpha+1}\right]^{p+1}}{\int_{\Omega} \varphi_{1}^{2}}$ is a positive number and condition $(\alpha p)_{1}$ is equivalent to $2-(\alpha+1)(p+1)>0$.

The main result of this section is as follows:

Theorem 2.1 Let $f \in C^{0}[0, \infty) \cap C^{1}(0, \infty)$ be a function satisfying $\left(f_{1}\right),\left(f_{2}\right),\left(F_{1}\right)$ and $(\alpha p)_{1}$. Then problem (1.2) possesses a positive solution $u_{1}$ satisfying $0<\left|u_{1}\right|_{\infty}<\theta_{1}$, whatever $\Omega$ bounded smooth domain. If, in addition, $f$ satisfies $\left(F_{2}\right)$ and $\Omega$ contains and it is near a ball $B_{R}$, with radius $R$ sufficiently large, problem (1.2) possesses a second solution $u_{2}$ satisfying

$$
0<\left|u_{1}\right|_{\infty}<\theta_{1}<\left|u_{2}\right|_{\infty}<\theta_{2}
$$

A standard bootstrap argument shows that such solutions are classical.

As we have said before, the term $\Omega$ contains and it is near a ball $B_{R}$, with radius $R$ sufficiently large will be precisely established in the proof of the second solution.

It is worthwhile to say that even in case $\Omega=B_{R}$ we may not infer that the solutions obtained are radial. We recall that in [23] the function $f$ should be of class $C^{1}[0,+\infty)$ in order to obtain radial symmetry. As it is well known, if we consider the problem

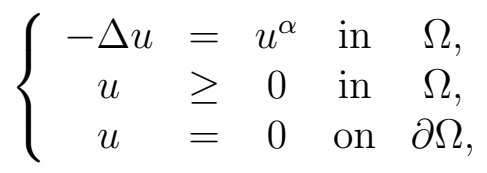

with $0<\alpha<1$, then nonradial solution may appear.

We will start by establishing a basic and simple lemma which will play a key role in the proof of theorem 3.1. 
Lemma 2.1 Let $f \in C^{0}[0, \infty) \cap C^{1}(0, \infty)$ be a function such that $f(\theta) \leq 0$, for some $\theta>0$, and $f$ satisfies $\left(F_{1}\right)$. Then problem (1.2) does not possess a classical positive solution $u$ with $|u|_{\infty}=\theta$.

Proof. Suppose, on the contrary, that $u \in C^{2}(\Omega) \cap C(\bar{\Omega})$ is a positive solution of (1.2), that is,

$$
\left\{\begin{array}{rlr}
-\Delta u & =f(u)\left[\int_{\Omega} F(u)\right]^{p} & \text { in } \quad \Omega \\
u & =0 & \text { on } \partial \Omega,
\end{array}\right.
$$

with $|u|_{\infty}=\theta$. Consequently, $0<u(x) \leq \theta$ for all $x \in \Omega$ and $|\{x \in \Omega ; u(x)<\theta\}|>0$. Let $M>0$ be such that $f(t)+M t \geq 0$ is increasing on $[0, \theta]$. Since

$$
\left\{\begin{aligned}
-\Delta u+M\left[\int_{\Omega} F(u)\right]^{p} u & =\left[\int_{\Omega} F(u)\right]^{p}[f(u)+M u] & \text { in } \Omega \\
u & =0 & \text { on } \partial \Omega
\end{aligned}\right.
$$

and

$$
\left\{\begin{aligned}
-\Delta \theta+M\left[\int_{\Omega} F(u)\right]^{p} \theta & \geq\left[\int_{\Omega} F(u)\right]^{p}[f(\theta)+M \theta] & \text { in } \Omega, \\
\theta & >0 & \text { on } \partial \Omega
\end{aligned}\right.
$$

we obtain

$$
\left\{\begin{array}{c}
-\Delta(\theta-u)+M\left[\int_{\Omega} F(u)\right]^{p}(\theta-u)= \\
{\left[\int_{\Omega} F(u)\right]^{p}[f(\theta)+M \theta-(f(u)+M u)] \geq 0 \text { in } \Omega,} \\
\theta-u>0 \text { on } \partial \Omega .
\end{array}\right.
$$

By the maximum principle, $\theta-u(x)>0$ on $\bar{\Omega}$ and so $|u|_{\infty}<\theta$ which contradicts our assumption. This proves the lemma.

Proof of the Theorem 2.1. First of all let us consider the problem

$$
\left\{\begin{array}{rlrl}
-\Delta u & =f_{1}(u)\left[\int_{\Omega} F_{1}(u)\right]^{p} & \text { in } & \Omega, \\
u & =0 & \text { on } \partial \Omega,
\end{array}\right.
$$


where $f_{1}: \mathbb{R} \rightarrow \mathbb{R}$ is the Hölder-continuous function defined by

$$
f_{1}(t)=\left\{\begin{array}{ccc}
f(0) & \text { if } & t \leq 0 \\
f(t) & \text { if } & 0<t \leq \theta_{1} \\
0 & \text { if } & t>\theta_{1}
\end{array}\right.
$$

where $F_{1}(t)=\int_{0}^{t} f_{1}(s) d s$.

We will find a solution of the problem (2.3) as a critical point of the functional $J_{1}: H_{0}^{1}(\Omega) \rightarrow \mathbb{R}$ given by

$$
J_{1}(u)=\frac{1}{2} \int_{\Omega}|\nabla u|^{2}-\frac{1}{p+1}\left[\int_{\Omega} F_{1}(u)\right]^{p+1}, u \in H_{0}^{1}(\Omega),
$$

where in $H_{0}^{1}(\Omega)$ we are considering the usual norm $\|u\|=\left(\int_{\Omega}|\nabla u|^{2}\right)^{\frac{1}{2}}$.

In view of the definition of $f_{1}$, we have $\int_{\Omega} F_{1}(u) \geq 0$ for all $u \in H_{0}^{1}(\Omega)$ and so $\left[\int_{\Omega} F_{1}(u)\right]^{p}$ and $\left[\int_{\Omega} F_{1}(u)\right]^{p+1}$ are well defined. Also, $\int_{\Omega} F_{1}(u) \leq$ $F_{1}\left(\theta_{1}\right)|\Omega|$ which implies that

$$
J_{1}(u) \geq \frac{1}{2} \int_{\Omega}|\nabla u|^{2}-\frac{1}{p+1}\left[F_{1}\left(\theta_{1}\right)|\Omega|\right]^{p+1}
$$

and so $J_{1}$ is bounded from below. Besides of this, it is a standard matter to show that $J_{1}$ is weakly lower semicontinuous and belongs to $C^{1}\left(H_{0}^{1}(\Omega), \mathbb{R}\right)$ with

$$
<J_{1}^{\prime}(u), \varphi>=\int_{\Omega} \nabla u \nabla \varphi-\left[\int_{\Omega} F_{1}(u)\right]^{p} \int_{\Omega} f_{1}(u) \varphi, \quad \forall u, \varphi \in H_{0}^{1}(\Omega) .
$$

Hence $J_{1}$ attains a minimum at $u_{1} \in H_{0}^{1}(\Omega)$ which is a weak solution of (2.3).

If $f(0)>0$ the solution $u_{1} \not \equiv 0$. In case $f(0)=0$, we should show that $u_{1} \not \equiv 0$ in $\Omega$. For this it is enough to show that $J_{1}\left(u_{1}\right)<0$. Let $\varphi_{1}>0$ an eigenfunction of $\left(-\Delta, H_{0}^{1}(\Omega)\right)$ satisfying $(\alpha p)_{2}$. Thus, taking $\epsilon>0$ such that 
$0<\epsilon \varphi_{1}(x)<\theta_{1}$, we obtain

$$
J_{1}\left(\epsilon \varphi_{1}\right)=\frac{1}{2} \epsilon^{2}\left\|\varphi_{1}\right\|^{2}-\frac{1}{p+1}\left[\int_{\Omega} F\left(\epsilon \varphi_{1}\right)\right]^{p+1},
$$

where $F(t)=\int_{0}^{t} f(s) d s$.

We now use condition $\left(f_{1}\right)$ to obtain $0<t_{0}<\theta_{1}$ and $\gamma>0$ such that

$$
\frac{f(t)}{t^{\alpha}} \geq \gamma \text { if } 0<t<t_{0}
$$

Then we choose $\epsilon>0$ such that $0<\epsilon \varphi_{1}(x)<t_{0}$ to obtain (because $\left.F\left(\epsilon \varphi_{1}\right)=F_{1}\left(\epsilon \varphi_{1}\right)\right)$

$$
\begin{gathered}
J_{1}\left(\epsilon \varphi_{1}\right)=\frac{1}{2} \epsilon^{2}\left\|\varphi_{1}\right\|^{2}-\frac{1}{p+1}\left[\int_{\Omega} F\left(\epsilon \varphi_{1}\right)\right]^{p+1} \leq \\
\frac{1}{2} \epsilon^{2}\left\|\varphi_{1}\right\|^{2}-\frac{1}{p+1}\left(\frac{\gamma}{\alpha+1}\right)^{p+1} \epsilon^{(\alpha+1)(p+1)}\left[\int_{\Omega} \varphi_{1}^{\alpha+1}\right]^{p+1} .
\end{gathered}
$$

Consequently,

$J_{1}\left(\epsilon \varphi_{1}\right) \leq \epsilon^{(\alpha+1)(p+1)}\left[\frac{1}{2} \epsilon^{2-(\alpha+1)(p+1)}\left\|\varphi_{1}\right\|^{2}-\frac{1}{p+1}\left(\frac{\gamma}{\alpha+1}\right)^{p+1}\left[\int_{\Omega} \varphi_{1}^{\alpha+1}\right]^{p+1}\right]$

and for $\epsilon>0$ small enough we have $J_{1}\left(\epsilon \varphi_{1}\right)<0$.

In this way, we have shown that $u_{1} \not \equiv 0$. Furthermore,

$$
-\Delta u_{1}=f_{1}\left(u_{1}\right)\left[\int_{\Omega} F_{1}\left(u_{1}\right)\right]^{p} \geq 0
$$

implies that $u_{1} \geq 0$ and because $u_{1} \not \equiv 0$ we conclude that $u_{1}>0$ in $\Omega$.

By Lemma 3.1, $u_{1}<\theta_{1}$ in $\Omega$ and so $0<u_{1}(x)<\theta_{1}$ for all $x \in \Omega$. Consequently, $u_{1}$ is a solution of the former problem (1.2). Moreover, for a future use, we remark that if $u \in H_{0}^{1}(\Omega), 0<u(x)<\theta_{1}$, one obtains

$$
J_{1}(u)>-\frac{1}{p+1}\left[\int_{\Omega} F(u)\right]^{p+1} .
$$


Indeed, if $u \in H_{0}^{1}(\Omega), 0<u(x)<\theta_{1}$ we have $F(u(x))<F\left(\theta_{1}\right)$ and so

$$
\int_{\Omega} F(u)<F\left(\theta_{1}\right)|\Omega|
$$

Thus,

$$
J_{1}(u)>-\frac{1}{p+1}\left[F\left(\theta_{1}\right)\right]^{p+1}|\Omega|^{(p+1)}
$$

for all $u \in H_{0}^{1}(\Omega)$ with $0 \leq u(x) \leq \theta_{1}$.

It is clear that this first solution may be obtained by another method as, for example, fixed point theory, including the case in which the problem is not variational. However, we would like to use solely variational method in order to obtain multiple solutions. In this way, (2.6) will play a key role.

Another fact it is worthy to recall is that the existence of $u_{1}$ does not depend on the size of $\Omega$. The size of $\Omega$ is essential for the existence of the second solution whose existence is shown by using a device motivated by Klaasen-Mitidieri [25].

For this we should consider the truncation $f_{2}: \mathbb{R} \rightarrow \mathbb{R}$ given by

$$
f_{2}(t)=\left\{\begin{array}{ccc}
f(0) & \text { if } & t<0 \\
f(t) & \text { if } & 0 \leq t \leq \theta_{3} \\
0 & \text { if } & t>\theta_{3}
\end{array}\right.
$$

and the corresponding problem

$$
\left\{\begin{aligned}
-\Delta u & =f_{2}(u)\left[\int_{\Omega} F_{2}(u)\right]^{p} & \text { in } \quad & \Omega \\
u & =0 & \text { on } & \partial \Omega
\end{aligned}\right.
$$

with $F_{2}(t)=\int_{0}^{t} f_{2}(s) d s$

As we performed to obtain the first solution, we find a minimizer $u_{2} \in$ $H_{0}^{1}(\Omega)$ to the functional

$$
J_{2}(u)=\frac{1}{2} \int_{\Omega}|\nabla u|^{2}-\frac{1}{p}\left[\int_{\Omega} F_{2}(u)\right]^{p+1}, \quad u \in H_{0}^{1}(\Omega) .
$$


Such a minimizer is a weak solution of the problem (2.7) satisfying $0<$ $u_{2}(x)<\theta_{2}$. We have to point out that $u_{2}$ is positive because $f_{2}(t)+M t$ is positive for some $M>0$ and for all $t \in \mathbb{R}$. So, $u_{2}$ is a solution of (1.2).

However, we can not affirm, up to now, that $u_{1} \neq u_{2}$. For this, we consider the function $u_{R} \in H_{0}^{1}(\Omega)$ defined by

$$
u_{R}(x)=\left\{\begin{array}{ccc}
\theta_{3} & \text { if } & |x| \leq R-1, \\
(R-|x|) \theta_{3} & \text { if } & R-1<|x|<R, \\
0 & \text { if } & x \in \Omega \backslash B_{R}
\end{array}\right.
$$

and set

$$
C_{R}=\left\{x \in \mathbb{R}^{N} ; R-1<|x|<R\right\}
$$

Thus

$$
\begin{gathered}
J_{2}\left(u_{R}\right)=\frac{1}{2} \int_{C_{R}}\left|\nabla u_{R}\right|^{2}-\frac{1}{p+1}\left[\int_{B_{R-1}} F\left(\theta_{3}\right)+\int_{C_{R}} F\left(u_{R}\right)\right]^{p+1} \\
J_{2}\left(u_{R}\right)=\frac{1}{2} \int_{C_{R}}\left|\nabla u_{R}\right|^{2}-\frac{1}{p+1}\left[F\left(\theta_{3}\right)\left|B_{R-1}\right|+\int_{C_{R}} F\left(u_{R}\right)\right]^{p+1} \\
J_{2}\left(u_{R}\right)=\frac{\theta_{3}^{2}}{2}\left|C_{R}\right|-\frac{1}{p+1}\left[F\left(\theta_{3}\right)\left|B_{R-1}\right|+\int_{C_{R}} F\left(u_{R}\right)\right]^{p+1} .
\end{gathered}
$$

Using

$$
\begin{gathered}
{\left[F\left(\theta_{3}\right)\left|B_{R-1}\right|+\int_{C_{R}} F\left(u_{R}\right)\right]^{p+1} \geq\left(F\left(\theta_{3}\right)\left|B_{R-1}\right|\right)^{p+1}+\left[\int_{C_{R}} F\left(u_{R}\right)\right]^{p+1}} \\
\Downarrow \\
J_{2}\left(u_{R}\right) \leq \frac{\theta_{3}^{2}}{2}\left|C_{R}\right|-\frac{1}{p+1}\left[F\left(\theta_{3}\right)\right]^{p+1}\left|B_{R-1}\right|^{p+1}+\frac{1}{p+1}\left[\int_{C_{R}} F\left(u_{R}\right)\right]^{p+1} \\
\Downarrow \\
J_{2}\left(u_{R}\right) \leq \frac{\theta_{3}^{2}}{2} h_{N} R^{N-1}-\frac{1}{p+1}\left[F\left(\theta_{3}\right)\right]^{p+1} K_{N}^{p+1}(R-1)^{N(p+1)}+C_{1} h_{N}^{p+1} R^{(N-1)(p+1)} .
\end{gathered}
$$

If $R$ is taken as in condition (2.1) we obtain

$$
J_{2}\left(u_{R}\right) \leq C_{N} R^{(N-1)(p+1)}-K_{N}^{p+1} \frac{\left[F\left(\theta_{3}\right)\right]^{p+1}}{p+1}(R-1)^{N(p+1)} .
$$


We would like to show that

$$
C_{N} R^{(N-1)(p+1)}-K_{N}^{p+1} \frac{\left[F\left(\theta_{3}\right)\right]^{p+1}}{p+1}(R-1)^{N(p+1)}<-\frac{1}{p+1}\left[F\left(\theta_{1}\right)\right]^{p+1}|\Omega|^{p+1} .
$$

Before to continue, we remember that $N(p+1)-(N-1)(p+1)=p+1$ and, by virtue of $\left(F_{2}\right),\left[F\left(\theta_{3}\right)\right]^{p+1}>\left[F\left(\theta_{1}\right)\right]^{p+1}$.

It is worthy to remark that all the calculations made up to now are valid for all $B_{R} \subset \Omega, R>1$.

Here, we note that condition $\left(F_{2}\right)$ implies that for each $r>0$, there exists $\bar{R}=\overline{R(r)}>0$ such that

$C_{N} R^{(N-1)(p+1)}-\frac{K_{N}^{p+1}}{p+1}\left[\left(F\left(\theta_{3}\right)\right)^{p+1}(R-1)^{N(p+1)}-\left(F\left(\theta_{1}\right)\right)^{p+1} R^{N(p+1)}\right]<-r$,

for $R>\bar{R}$. Let us fix some $R$ like this and remember that $\left|B_{R}\right|=K_{N} R^{N}$. So,

$C_{N} R^{(N-1)(p+1)}-\frac{K_{N}^{p+1}}{p+1}\left(F\left(\theta_{3}\right)\right)^{p+1}(R-1)^{N(p+1)}<-r-\frac{1}{p+1}\left(F\left(\theta_{1}\right)\right)^{p+1}\left|B_{R}\right|^{p+1}$

and choose $\Omega$ such that $B_{R} \subset \Omega$ and

$$
\frac{1}{p+1}\left(F\left(\theta_{1}\right)\right)^{p+1}\left[|\Omega|^{p+1}-\left|B_{R}\right|^{p+1}\right]<r .
$$

This is the precise meaning of the expression contains and it is near a ball $B_{R}$.

Thus,

$$
J_{2}\left(u_{2}\right) \leq J_{2}\left(u_{R}\right)<-\frac{1}{p+1}\left(F\left(\theta_{1}\right)\right)^{p+1}|\Omega|^{p+1}<J_{1}\left(u_{1}\right)=J_{2}\left(u_{1}\right) .
$$

This shows that $u_{1} \neq u_{2}$ and the proof of the theorem is over.

Remark 2.1 We should emphasize that, in the previous theorem, if $f(0)>0$ we may suppose that $f$ is of $C^{1}$-class on $[0, \infty)$. So, under this assumption and if $\Omega=B_{R}$, the obtained solutions are radial and decreasing with respect to $r=|x|$. 
Remark 2.2 As we have shown, $u_{1}$ is a minimum of $J_{1}$ and $u_{2}$ is a minimum of $J_{2}$. We conjecture that $u_{1}$ is also a minimum for $J_{2}$. If this is the case, an application of the Mountain Pass Theorem would lead us to a third solution $u_{3}$. However, we were not able to prove this. We also conjecture that $u_{1}$ and $u_{2}$ are ordered, i.e., $0<u_{1}(x)<u_{2}(x)$ for all $x \in \Omega$.

Remark 2.3 As we have said before, if $f(0)=0$, then $f_{+}^{\prime}(0)=+\infty$ which combined with the fact that $f(t)>0$, for $0<t<\theta_{1}$ and $f\left(\theta_{1}\right)=0$ implies that the graph of $f$ crosses the graph of the straight line $\lambda_{1}(\Omega) t$ for some $0<t<\theta_{1}$. It seems that this crossing produces the first solution $u_{1}$. However, the existence of positive solution fails if $f(0)=0$ and $\left(f_{1}\right)$ does not hold. To check this, let us suppose that the function $f$ satisfies $0 \leq f(t) \leq m t$, for all $t \geq 0,0 \leq g(t) \leq C$ and $u$ is a positive solution of (1.1).

A simple calculations leads us to

$$
0<\lambda_{1}(\Omega) \leq C|\Omega|^{p}
$$

where $C>0$ is a positive constant does not depend on $\Omega$. This is a contradiction because $\lambda_{1}(\Omega) \rightarrow+\infty$ as $|\Omega| \rightarrow 0$. The equation below

$$
\left\{\begin{array}{rlrl}
-\Delta u & =\sin u\left[\int_{\Omega} g(u)\right]^{2 p} & \text { in } \quad & \\
u & =0 & \text { on } \partial \Omega
\end{array}\right.
$$

with $0 \leq g(t) \leq C$ satisfies these conditions.

Example 2.1 In case $f(0)>0$, the function $f(t)=-t^{3}+(3+a) t^{2}-(3 a+$ $2) t+2 a, t \geq 0$, enjoys the assumptions of Theorem 2.1 in which the positive zeroes of $f$ are $\frac{4}{10}=a<1<2$. Here whatever $p, 0<p<1$, is valid because we can choose $\alpha$ verifying $(\alpha p)_{1}$ and $f_{1}$.

Example 2.2 When $f(0)=0$ we may consider the function $f:[0, \infty) \rightarrow \mathbb{R}$ defined by

$$
f(t)=t^{\alpha}(t-a)(t-1) \text { for } 0 \leq t \leq 1
$$




$$
f(t)=-(1-a) t^{2}+3(1-a) t-2(1-a) \text { for } t \geq 1
$$

where $0<\alpha<1$ and $\frac{\alpha+1}{\alpha+3}<a<1$. Such a function satisfies the assumptions of the aforementioned result.

\section{$3 f(0)=0$ and $f<0$ near 0}

In this section we consider the problem

$$
\left\{\begin{aligned}
-\Delta u & =f(u)\left[\int_{\Omega} F(u)\right]^{2 p} & \text { in } \quad \Omega \\
u & =0 & \text { on } \partial \Omega
\end{aligned}\right.
$$

with $p=0,1,2, \ldots$ and $f \in C^{1}[0, \infty)$ satisfies

$\left(f_{3}\right) \quad\left\{\begin{array}{l}f(0)=0 \text { and there are } 0<a_{1}<a_{2} \\ \text { such that } f\left(a_{1}\right)=f\left(a_{2}\right)=0, \\ f(t)<0 \text { if } 0<t<a_{1} \text { and } f(t)>0 \text { if } a_{1}<t<a_{2} .\end{array}\right.$

$$
F\left(a_{2}\right)>0, \text { where } F(t)=\int_{0}^{t} f(s) d s .
$$

In view of condition $\left(F_{3}\right)$, there is $0<a_{3}<a_{2}$ such that $F\left(a_{3}\right)=0, F(t)<0$ if $0<t<a_{3}$ and $F(t)>0$ if $a_{3}<t<a_{2}$.

From now on, we still denote by $f$ the extension-truncation of $f$ such that $f(t)=f(0)=0$ if $t<0$ and $f(t)=0$ if $t>a_{2}$.

Let $J: H_{0}^{1}(\Omega) \rightarrow \mathbb{R}$ be the energy functional associated to problem (3.1) given by

$$
J(u)=\frac{1}{2}\|u\|^{2}-\frac{1}{2 p+1}\left[\int_{\Omega} F(u)\right]^{2 p+1}
$$

where, as before, $\|\cdot\|$ is the usual norm of $H_{0}^{1}(\Omega)$.

Theorem 3.1 Under assumptions $\left(f_{3}\right)-\left(F_{3}\right)$ and if $\Omega$ contains a ball $B_{R}$ with $R$ large enough, problem (3.1) possesses two positive solutions satisfying $a_{1}<\left|u_{1}\right|_{\infty},\left|u_{2}\right|_{\infty}<a_{2}$. Furthermore, if $\Omega=B_{R}$ such solutions are radial and $\frac{\partial u_{i}}{\partial r}<0$ for $0<r<R$ and $i=1,2$. 
Proof. As in the previous result, we may show that $J$ is coercive, bounded from below, of $C^{1}$-class and so on. Hence $J$ attains a global minimum at a certain $u_{1} \in H_{0}^{1}(\Omega)$. Since $f(0)=0$ we have to show that $u_{1} \not \equiv 0$. For this, we consider, like before, the function

$$
u_{R}(x)=\left\{\begin{array}{ccc}
a_{2} & \text { if } & |x| \leq R-1 \\
(R-|x|) a_{2} & \text { if } & R-1<|x|<R \\
0 & \text { if } & x \in \Omega \backslash B_{R}
\end{array}\right.
$$

Let us evaluate $J\left(u_{R}\right)$ : Observing that $0<u_{R}(x) \leq a_{2}$ for all $x \in \Omega$, we obtain

$$
J\left(u_{R}\right)=\frac{1}{2} a_{2}^{2}\left|C_{R}\right|-\frac{1}{2 p+1}\left[F\left(a_{2}\right)\left|B_{R-1}\right|+\int_{C_{R}} F\left(u_{R}\right)\right]^{2 p+1}
$$

where $C_{R}=\left\{x \in \mathbb{R}^{N} ; R-1<|x|<R\right\}$. We now note that $F\left(u_{R}(x)\right) \geq$ $\min _{0 \leq t \leq a_{2}} F(t)=F\left(a_{1}\right)$ to obtain $\int_{C_{R}} F\left(u_{R}(x)\right) d x \geq F\left(a_{1}\right)\left|C_{R}\right|$ and so

$$
F\left(a_{2}\right)\left|B_{R-1}\right|+\int_{C_{R}} F\left(u_{R}\right) \geq F\left(a_{2}\right)\left|B_{R-1}\right|+F\left(a_{1}\right)\left|C_{R}\right|
$$

and, because the function $t \rightarrow t^{2 p+1}, t \in \mathbb{R}$ is increasing,

$$
\left[F\left(a_{2}\right)\left|B_{R-1}\right|+\int_{C_{R}} F\left(u_{R}\right)\right]^{2 p+1} \geq\left[F\left(a_{2}\right)\left|B_{R-1}\right|+F\left(a_{1}\right)\left|C_{R}\right|\right]^{2 p+1} .
$$

Consequently,

$$
J\left(u_{R}\right) \leq \frac{1}{2} a_{2}^{2}\left|C_{R}\right|-\frac{1}{2 p+1}\left[F\left(a_{2}\right)\left|B_{R-1}\right|+F\left(a_{1}\right)\left|C_{R}\right|\right]^{2 p+1} .
$$

We now point out that:

- $\frac{1}{2} a_{2}^{2}\left|C_{R}\right|$ behaves like $\frac{1}{2} a_{2}^{2} R^{N-1}$ at infinity;

- $F\left(a_{2}\right)\left|B_{R-1}\right|$ behaves like $F\left(a_{2}\right)(R-1)^{N}$ at infinity and recall that $F\left(a_{2}\right)>0$

- $F\left(a_{1}\right)\left|C_{R}\right|$ behaves like $F\left(a_{1}\right) R^{N-1}$ at infinity. 
Thus $\left(F\left(a_{2}\right)\left|B_{R-1}\right|+F\left(a_{1}\right)\left|C_{R}\right|\right)^{2 p+1}$ behaves like $F\left(a_{2}\right)^{2 p+1} R^{N(2 p+1)}$ at infinity and so

$$
J\left(u_{R}\right)<0 \text { if } R \text { is large enough. }
$$

From now on, we fix such a large $R$. Consequently, $J\left(u_{1}\right) \leq J\left(u_{R}\right)<0$ and so $u_{1} \not \equiv 0$. Let $u^{-}$be the negative part of $u=u^{+}-u^{-}$. Multiplying both sides of

$$
\left\{\begin{aligned}
-\Delta u_{1} & =f\left(u_{1}\right)\left[\int_{\Omega} F\left(u_{1}\right)\right]^{2 p} & \text { in } \quad \Omega, \\
u_{1} & =0 & \text { on } \partial \Omega,
\end{aligned}\right.
$$

by $u_{1}^{-}$and integrating by parts, we obtain

$$
-\left\|u_{1}^{-}\right\|^{2}=\left[\int_{\Omega} F\left(u_{1}\right)\right]^{2 p} \int_{\Omega} f\left(u_{1}\right) u_{1}^{-}=0 .
$$

Hence, $u_{1} \geq 0$ in $\Omega$ and reasoning as in Lemma 2.1 and in view of the maximum principle we conclude that $0<u_{1}(x)<a_{2}$ and $a_{1}<\left|u_{1}\right|_{\infty}<a_{2}$.

We now claim that 0 is also a local minimum of $J$. Indeed, if $t \geq 0$ we have $f(t) \leq \alpha t$, for some $\alpha>0$, and so $F(t) \leq \frac{\alpha}{2} t^{2}$ for all $t \geq 0$. Since $F(t)=0$ if $t<0$, this inequality remains true for all $t \in \mathbb{R}$. Hence, $\int_{\Omega} F(u) \leq C\|u\|^{2}$ from which $\left[\int_{\Omega} F(u)\right]^{2 p+1} \leq C\|u\|^{2(2 p+1)}$. Consequently,

$$
J(u) \geq \frac{1}{2}\|u\|^{2}-C\|u\|^{2(2 p+1)}=\|u\|^{2}\left(\frac{1}{2}-C\|u\|^{4 p}\right)>0
$$

if $\|u\|=\rho>0, \rho$ small enough. This shows that 0 is a strict local minimum.

We now use the well known Mountain Pass Theorem due to AmbrosettiRabinowitz [1]. For this we recall the following concepts:

We say that the functional of class $C^{1}, I: X \rightarrow \mathbb{R}, X$ is a Banach space, satisfies the Palais-Smale condition $(P S)$ if any sequence $\left(u_{n}\right) \subset X$ such that $\left|I\left(u_{n}\right)\right| \leq C$ and $I^{\prime}\left(u_{n}\right) \rightarrow 0$ in $X^{*}$ possesses a convergent subsequence.

(Mountain Pass Theorem) Let $X$ be a reflexive Banach space and $I: X \rightarrow \mathbb{R}$ a functional of class $C^{1}$ satisfying the Palais-Smale condition and the following geometric conditions: 
(i) $I(0)=0$ and there exist $\rho, \alpha>0$ such that $I(u) \geq \alpha$ if $\|u\|=\rho$ where $\|\cdot\|$ is the norm in $X$;

(ii) there exists $e \in X$ such that $\|e\|>\rho$ and $I(e) \leq 0$.

Then $I$ has a critical value $c \geq \alpha$ given by

$$
c=\inf _{\gamma \in \Gamma} \sup _{t \in[0,1]} I(\gamma(t))
$$

where $\Gamma=\{\gamma \in C([0,1]) ; \gamma(0)=0, \quad \gamma(1)=e\}$.

Combining the fact that

$$
J\left(u_{R}\right)<0 \text {, if } R \text { is fixed and large enough, }
$$

with

$$
J(u) \geq \frac{1}{2}\|u\|^{2}-C\|u\|^{2(2 p+1)}=\|u\|^{2}\left(\frac{1}{2}-C\|u\|^{4 p}\right)>0
$$

we find $\rho, \alpha$, with $\rho>0$ small enough, so that $e=u_{R}$ and $\|e\|=\left\|u_{R}\right\|>\rho$ and so $J$ satisfies the geometry of the Mountain Pass Theorem.

For the sake of completeness we show that $J$ enjoys the $(P S)$ condition. For this, let $\left(u_{n}\right) \subset H_{0}^{1}(\Omega)$ such that $\left|J\left(u_{n}\right)\right| \leq C$ and $J^{\prime}\left(u_{n}\right) \rightarrow 0$ in $\left(H_{0}^{1}(\Omega)\right)^{*}=H^{-1}(\Omega)$. Since $J$ is coercive, it follows that $\left(u_{n}\right)$ is bounded in $H_{0}^{1}(\Omega)$ and so $u_{n} \rightarrow u$, weakly in $H_{0}^{1}(\Omega), u_{n} \rightarrow u$ in $L^{r}(\Omega)$ in $L^{r}(\Omega), 1 \leq$ $r \leq 2^{*}$, where $2^{*}$ is the critical Sobolev exponent, and $u_{n}(x) \rightarrow u(x)$ a.e. in $\Omega$, perhaps for subsequences.

Furthermore,

$$
<J^{\prime}(u), v>=\int_{\Omega} \nabla u \nabla v-\left[\int_{\Omega} F(u)\right]^{2 p} \int_{\Omega} f(u) v, \text { for all } u, v \in H_{0}^{1}(\Omega)
$$

and so

$$
<\nabla J(u), v>=\left\langle u, v>-<T u, v>\text { for all } u, v \in H_{0}^{1}(\Omega)\right.
$$


where $<,>$ is the usual inner product in $H_{0}^{1}(\Omega), \nabla J(u)$ is the gradient of $J$ in $u \in H_{0}^{1}(\Omega)$ and $T: H_{0}^{1}(\Omega) \rightarrow H_{0}^{1}(\Omega)$ defined by

$$
<T u, v>=\left[\int_{\Omega} F(u)\right]^{2 p} \int_{\Omega} f(u) v, \text { for all } u, v \in H_{0}^{1}(\Omega)
$$

and clearly is compact. Hence $\nabla J(u)=u-T u$ and because $\nabla J\left(u_{n}\right) \rightarrow 0$ in $H_{0}^{1}(\Omega)$ and, in view of compactness of $T,\left(T\left(u_{n}\right)\right)$ is convergent in $H_{0}^{1}(\Omega)$, perhaps for a subsequence, we conclude that $u_{n} \rightarrow u$ in $H_{0}^{1}(\Omega)$ up to a subsequence. This shows that $J$ satisfies the assumptions of the Mountain Pass Theorem and so problem (3.1) possesses a second solution $u_{2}$.

To finish the proof we have to point out that both positive solutions we have found are classical and satisfy $a_{1}<\left|u_{1}\right|_{\infty},\left|u_{2}\right|_{\infty}<a_{2}$. In case we are working in a ball, both $u_{1}, u_{2}$ are radial and $\frac{\partial u_{1}}{\partial r}, \frac{\partial u_{2}}{\partial r}<0$ if $0<r<R$.

Remark 3.1 Let $u$ be a solution of problem (3.1) with $0<u(x)<a_{2}$. Thus

$$
\|u\|^{2}=\left[\int_{\Omega} F(u)\right]^{2 p} \int_{\Omega} f(u) u
$$

and because

$$
\|u\|^{2}=\left[\int_{\Omega} F(u)\right]^{2 p}\left[\int_{0<u(x) \leq a_{1}} f(u) u+\int_{a_{1}<u(x) \leq a_{2}} f(u) u\right] \leq\|u\|^{2}
$$

we obtain

$$
\|u\|^{2} \leq\left[\int_{\Omega} F(u)\right]^{2 p} \int_{a_{1}<u(x) \leq a_{2}} f(u) u
$$

because $f(t)<0$ for $0 \leq t<a_{1}$. Since $f$ is bounded, we may find a constante $C>0$ such that $f(t) t \leq C\left(1+t^{2}\right)$ which yields

$$
\|u\|^{2} \leq C\left[\int_{\Omega} F(u)\right]^{2 p} \int_{a_{1}<u(x) \leq a_{2}}\left(1+u^{2}\right) .
$$

Since $\frac{u(x)}{a_{1}}>1$, we obtain

$$
\|u\|^{2} \leq C_{1}\left[\int_{\Omega} F(u)\right]^{2 p} \int_{a_{1}<u(x) \leq a_{2}} u^{2} \leq C_{2}\left[\int_{\Omega} F(u)\right]^{2 p} \int_{\Omega} u^{2} .
$$


We now use the variational characterization of $\lambda_{1}=\lambda_{1}(\Omega)$, and recalling that $\Omega=B_{R}$, to get

$$
\|u\|^{2} \leq C\left[F\left(a_{2}\right)\right]^{2 p} \frac{|\Omega|^{2 p}}{\lambda_{1}(\Omega)}\|u\|^{2}
$$

and so

$$
0<C \leq \frac{\left|B_{R}\right|^{2 p}}{\lambda_{1}(R)} \rightarrow 0 \text { as } R \rightarrow 0 .
$$

Since the constant $C$ does not depend on $\Omega$, we conclude that problem (3.1) does not posses solution if $R>0$ is small.

It follows from this remark and theorem 3.1 that there are $0<R_{0} \leq$ $R_{1}<\infty$ such that problem (3.1) has no solution if $R \in\left(0, R_{0}\right]$ and has two positive solutions if $R \in\left[R_{\infty}, \infty\right)$. Consequently, there is a range $\left[R_{0}, R_{\infty}\right]$ for which we were not able to determine existence of solution.

Remark 3.2 From condition $\left(F_{2}\right)$, there exists $a_{1}<a_{3}<a_{2}$ such that $F\left(a_{3}\right)=0, F(t)<0$ if $0<t<a_{3}$ and $F(t)>0$ if $a_{3}<t<a_{2}$. We conjecture that positive solutions $u$ of (3.1) should satisfy $a_{3}<|u|_{\infty}<a_{2}$.

Example 3.1 The function $f(t)=t(1-t)(t-a), 0<a<\frac{1}{2}$, which appears in the FitzHugh-Nagumo equation, satisfies the assumptions of the above result.

\section{The Semipositone Case}

In this section we study problem (3.1) under the semipositone point of view. More precisely, we will consider $f:[0, \infty) \rightarrow \mathbb{R}$ as a $C^{1}$-function satisfying

$$
f(0)=-a<0 .
$$

Beside of this we also suppose

$$
\left\{\begin{array}{l}
\text { There are } 0<b_{1}<b_{2} \text { such that } \\
f\left(b_{1}\right)=f\left(b_{2}\right)=0, f(t)<0 \text { if } 0<t<b_{1} \\
\text { and } f(t)>0 \text { if } b_{1}<t<b_{2}
\end{array}\right.
$$


and

$$
F\left(b_{2}\right)>0
$$

Semipositone local problems (former called nonpositone) appear in several physical situations as buckling of mechanical systems, design of suspension bridges, chemical reactions, astrophysics (thermal equilibrium of plasmas), combustion and management of natural resources. As it is said in CastroMaya-Shivaji [9], the difficulty of studying positive solutions to such problems was first encountered by Brown-Shivaji [6] when they studied the perturbed bifurcation problem

$$
-\Delta u=\lambda\left(u-u^{3}\right)-\epsilon \text { in } \Omega, u=0 \text { on } \Omega
$$

where $\epsilon>0$ is a given number. However, the study of semipositone problems was formally introduced by Castro-Shivaji [10]. For more informations on this kind of problem the interested reader may consult [9] and the references therein. With respect to nonlocal semipositone problems, at least to our knowledge, the present work is the first one.

Here, we attack problem (3.1), with $f(0)<0$, by using the device explored in Arcoya-Calahorrano [2]. However, we believe that the approach used in Costa-Tehrani-Yang [19], who use variational techniques for locally Lipschitz continuous functional like the one contained in Chang [12], also works in our present nonlocal problem.

In the context of semipositone problems we have to consider another concept of solution. For this, we consider the multivalued problem

$$
\left\{\begin{aligned}
-\Delta u & \in \hat{f}(u)\left[\int_{\Omega} \hat{F}(u)\right]^{2 p} & \text { a.e. in } & \Omega, \\
u & =0 & \text { on } & \partial \Omega
\end{aligned}\right.
$$

where $\hat{f}: \mathbb{R} \rightarrow 2^{\mathbb{R}}$ is the multivalued function defined by

$$
\hat{f}(t)=0 \text { if } t<0 ; \hat{f}(t)=[f(0), 0] \text { if } t=0 ; \hat{f}(t)=f(t) \text { if } t>0 .
$$


By a solution of (4.1) we mean a function $u \in C^{1}(\bar{\Omega}) \cap C^{2}\left(\Omega^{*}\right)$, with $\Omega^{*}=\{x \in \Omega ; u(x) \neq 0\}$, satisfying (4.1).

Note that, in view of boundedness of $f$ and elliptic regularity one has $u \in W^{2, p}(\Omega)$ for all $p \geq 1$. Consequently, $u \in C^{1, \alpha}(\bar{\Omega})$ for some $0<\alpha<1$.

We observe that every solution of (4.1) is nonnegative. Indeed, let us suppose, on the contrary, that the open set $\Omega_{-}=\{x \in \Omega ; u(x)<0\} \neq \emptyset$. Thus, if $\varphi \in C_{c}^{\infty}\left(\Omega_{-}\right)$, we obtain

$$
\int_{\Omega_{-}} \nabla u \nabla \varphi=0, \text { for all } \varphi \in C_{c}^{\infty}\left(\Omega_{-}\right)
$$

and so, by the maximum principle, $u=0$ a.e. in $\Omega_{-}$which is a contradiction. Consequently, $u \geq 0$ a.e. in $\Omega$. This implies that $u$ solves the problem

$$
\left\{\begin{aligned}
-\Delta u & \in \bar{f}(u)\left[\int_{\Omega} \bar{F}(u)\right]^{2 p} & \text { a.e. in } & \Omega \\
u & =0 & \text { on } & \partial \Omega
\end{aligned}\right.
$$

where $\bar{f}: \mathbb{R} \rightarrow 2^{\mathbb{R}}$ is the multivalued function defined by

$$
\bar{f}(t)= \begin{cases}{[f(0), 0]} & \text { if } t=0 \\ f(t) & \text { if } t>0\end{cases}
$$

and $\bar{F}(t)=\int_{0}^{t} \bar{f}(s) d s$

We should emphasize that, in the context of semipositone problems, we can not conclude that nonnegative solutions are, in fact, positive. In this way, the reader may consult Castro-Shivaji [10] in which is given an example of a semipositone problem where the solution is nonnegative and vanishes at some points. However, in our present problem we are able to show the positiveness of the solutions when we are dealing with a ball.

To attack problem problem (4.1) we consider the sequence of approximating problems like the one considered in the previous section

$$
\left\{\begin{aligned}
-\Delta u & =f_{n}(u)\left[\int_{\Omega} F_{n}(u)\right]^{2 p} & \text { in } \quad & \Omega, \\
u & =0 & \text { on } & \partial \Omega,
\end{aligned}\right.
$$


where $f_{n}: \mathbb{R} \rightarrow \mathbb{R}$ is the sequence of $C^{1}$-functions defined by

$$
f_{n}(t)=\left\{\begin{aligned}
f(t) & \text { if } t>\frac{1}{n} \\
0 & \text { if } \quad t \leq 0
\end{aligned}\right.
$$

with $f_{n}(t) \geq f(t)$ for $t \in\left(0, \frac{1}{n}\right]$ and $f_{n+1}(t) \leq f_{n}(t)$ for all $n \in \mathbb{I}$ and for all $t \in \mathbb{R}$. Hence, $F(t) \leq F_{n}(t)$ for all $t \geq 0$ and $F_{n+1}(t) \leq F_{n}(t)$ for all $t \geq 0$, where

$$
F_{n}(t)=\int_{0}^{t} f_{n}(s) d s
$$

and

$$
F(t)= \begin{cases}0 & \text { if } t<0, \\ \int_{0}^{t} f(s) d s & \text { if } t \geq 0 .\end{cases}
$$

From now on we fix $B_{R} \subset \Omega$, for $R>0$ large enough as in the previous section and consider the energy functional

$$
I_{n}(u)=\frac{1}{2} \int_{\Omega}|\nabla u|^{2}-\frac{1}{2 p+1}\left[\int_{\Omega} F_{n}(u)\right]^{2 p+1}
$$

associated to the problem (4.1).

Theorem 4.1 Under assumptions $\left(f_{4}\right)-\left(f_{5}\right)-\left(F_{4}\right)$ and $\Omega$ is as in Theorem 3.1 , problem (4.3) possesses at least two positive classical solutions $u_{n} \neq v_{n}$ with $\left\|u_{n}\right\|_{\infty},\left\|v_{n}\right\|_{\infty}>b_{1}$.

Proof. As it is easy to see, problem (4.3) satisfies, for all $n \in \mathbb{N}$ large enough, all the assumptions of theorem 3.1. Furthermore,

$$
\begin{gathered}
I_{n}\left(u_{R}\right)=\frac{1}{2} \int_{\Omega}\left|\nabla u_{R}\right|^{2}-\frac{1}{2 p+1}\left[\int_{\Omega} F_{n}\left(u_{R}\right)\right]^{2 p+1} \leq \\
\frac{1}{2} \int_{\Omega}\left|\nabla u_{R}\right|^{2}-\frac{1}{2 p+1}\left[\int_{\Omega} F\left(u_{R}\right)\right]^{2 p+1}< \\
\frac{1}{2} b_{2}^{2}\left|C_{R}\right|-\frac{1}{2 p+1}\left[F\left(b_{2}\right)\left|B_{R-1}\right|+F\left(b_{1}\right)\left|C_{R}\right|\right]^{2 p+1}<0,
\end{gathered}
$$

for $R>0$ large enough and fixed, where $u_{R}$ is the one introduced in sect. 3 , because $F_{n}(t) \geq F(t)$ for all $t \in \mathbb{R}$, where the last inequality is obtained 
like in the previous section and does not depend on $n \in \mathbb{N}$. Also, because $F_{n}(t) \leq F_{1}(t) \leq \alpha t^{2}$ for all $t \in \mathbb{R}$, and some $\alpha>0$, we obtain

$$
I_{n}(u) \geq \frac{1}{2}\|u\|^{2}-C(R)\|u\|^{4 p+2}=\|u\|^{2}\left(\frac{1}{2}-C(R)\|u\|^{4 p}\right)
$$

where $C(R)$ is a positive constant that depends on the fixed $R>0$ but does not depend on $n \in \mathbb{N}$. Consequently, there are positive constants $\rho=\rho(R), a=a(R)$ such that

$$
I_{n}(u) \geq a \text { if }\|u\|=\rho .
$$

Hence, by minimization and the Mountain Pass Theorem, like in the previous section, we obtain two positive solutions of problem (4.3) satisfying

$$
I_{n}\left(u_{n}\right) \leq \delta<0<a \leq I_{n}\left(v_{n}\right)
$$

where $\delta=\delta(R)$ does not depend on $n \in \mathbb{N}$.

It is clear that $b_{1}<\left|u_{n}\right|_{\infty},\left|v_{n}\right|_{\infty}<b_{2}$. This finishes the proof of this theorem.

In what follows, by a limiting process, we prove the existence of two nonnegative solutions for problem (4.1). The procedure is similar, mutatis mutandis, to that contained in [2]. However, for the sake of completness we will exhibit the details.

Theorem 4.2 Under the same assumptions of the Theorem 4.1, problem (4.2) possesses at least two nonnegative solutions. Furthermore, if $\Omega=B_{R}$ such solutions are radial and positive and so they solve (3.1).

Proof. Since $\left(u_{n}\right),\left(v_{n}\right)$ are bounded sequences in the uniform norm, the elliptic regularity theory shows that $\left(u_{n}\right),\left(v_{n}\right)$ are bounded in $W^{2, s}(\Omega)$ for all $s \geq 1$. So, up to a subsequence, we have $u_{n} \rightarrow u, v_{n} \rightarrow v$ in $C^{1, \nu}(\bar{\Omega})$ for some $0<\nu<1$. Consequently, $u, v \geq 0$ in $\Omega$ and, thanks to, $\left|u_{n}\right|_{\infty},\left|v_{n}\right|_{\infty}>b_{1}$, for all $n \in \mathbb{N}$, we have $|u|_{\infty},|v|_{\infty}>b_{1}$ and so $u, v \not \equiv 0$ in $\Omega$. 
Noticing that $F_{n}(t) \rightarrow F(t)$ in $\mathbb{R}$ and $u_{n} \rightarrow u, v_{n} \rightarrow v$ in $C^{1, \nu}(\bar{\Omega})$ for some $0<\nu<1$, we may use the Lebesgue Dominated Convergence Theorem to arrive in $\lim _{n \rightarrow \infty}\left\|u_{n}\right\|^{2}=\|u\|^{2}, \lim _{n \rightarrow \infty}\left\|v_{n}\right\|^{2}=\|v\|^{2}$ and $\lim _{n \rightarrow \infty} \int_{\Omega} F_{n}\left(u_{n}(x)\right)=$ $\lim _{n \rightarrow \infty} \int_{\Omega} F(u(x)), \quad \int_{\Omega} F_{n}\left(v_{n}(x)\right)=\lim _{n \rightarrow \infty} \int_{\Omega} F(v(x))$. Hence,

$$
\begin{gathered}
I(u) \equiv \frac{\|u\|^{2}}{2}-\frac{1}{2 p+1}\left[\int_{\Omega} F(u)\right]^{2 p+1} \leq \delta<0< \\
a<\frac{\|v\|^{2}}{2}-\frac{1}{2 p+1}\left[\int_{\Omega} F(v)\right]^{2 p+1} \equiv I(v)
\end{gathered}
$$

which shows that $u \neq v$.

In order to show that $u, v$ solve (4.2), we observe that $\lim _{n \rightarrow \infty} f_{n}\left(x_{n}\right)=f(x)$ if $\lim _{n \rightarrow \infty} x_{n}=x>0$ and $\lim _{n \rightarrow \infty} \int_{\Omega} F_{n}\left(u_{n}\right)=\int_{\Omega} F(u)$. Thus

$$
\Omega^{*}(u)=\{x \in \Omega ; u(x) \neq 0\}=\{x \in \Omega ; u(x)>0\} .
$$

Since $\Omega^{*}(u)$ is an open nonempty set we have

$$
\int_{\Omega^{*}(u)} \nabla u_{n} \cdot \nabla \varphi=\left[\int_{\Omega} F_{n}\left(u_{n}\right)\right]^{2 p} \int_{\Omega^{*}(u)} f_{n}\left(u_{n}(x)\right) \varphi, \quad \forall \varphi \in C_{c}^{\infty}\left(\Omega^{*}(u)\right) .
$$

Consequently, by virtue of the convergence $u_{n} \rightarrow u$ in $C^{1, \nu}(\bar{\Omega})$ we obtain

$$
\int_{\Omega^{*}(u)} \nabla u \nabla \varphi=\left[\int_{\Omega} F(u)\right]^{2 p} \int_{\Omega^{*}(u)} \bar{f}(u) \varphi, \forall \varphi \in C_{c}^{\infty}\left(\Omega^{*}(u)\right) .
$$

By the fundamental lemma of the calculus of variations

$$
-\Delta u(x)=f(u(x)) \text { in } \Omega^{*}(u)
$$

and by the elliptic regularity theory $u \in C^{2}\left(\Omega^{*}(u)\right)$. Using a well known result by Stampacchia [29]

$$
-\Delta u(x)=0 \text { in } \Omega / \Omega^{*}(u),
$$


that is, $-\Delta u(x) \in[-a, 0]=\bar{f}(0)$. Hence,

$$
\left\{\begin{aligned}
-\Delta u(x) & \in\left[\int_{\Omega} \bar{F}(u)\right]^{2 p} \bar{f}(u(x)) & \text { a.e. in } & \Omega, \\
u & =0 & \text { on } & \partial \Omega .
\end{aligned}\right.
$$

Reasoning as before, we may prove that $v$ is also a solution of (4.1).

In case $\Omega$ is a ball the approximating sequence of solutions satisfy $\left\|u_{n}\right\|_{\infty},\left\|v_{n}\right\|_{\infty}>b_{1}$ are radial and $\frac{\partial u_{n}}{\partial r}, \frac{\partial v_{n}}{\partial r}<0$ if $0<r<R$. Consequently, the limits $u, v$ are nonnegative, nonidentically zero and $\frac{\partial u}{\partial r}, \frac{\partial v}{\partial r} \leq 0,0<r<R$, which implies that both of them attains maximum at $0 \in B_{R}$. Consequently, there is $0<\rho \leq R$ such that $u(x)>0$ for $|x|<\rho$. Let us suppose that $\rho<R$. Since $u$ satisfies

$$
\left\{\begin{array}{ccccc}
-\Delta u & = & f(u)\left[\int_{\Omega} F(u)\right]^{2 p} & \text { in } & B_{\rho} \\
u & = & 0 & \text { on } & \partial B_{\rho}
\end{array}\right.
$$

and so, by the maximum principle, and because $u \in C^{1, \delta}(\bar{\Omega})$, for some $0<\delta<1$, it follows that $\frac{\partial u}{\partial r}(\rho)<0$. This contradicts with the fact that $\rho<R$ because, in this case, $u$ would attains a minimum at all $x$ with $|x|=\rho$. Consequently, we would have $\frac{\partial u}{\partial r}(\rho)=0$. Thus $u$ is positive in $B_{R}$ and solves (3.1). We reason in the same way for $v$.

Example 4.1 For the semipositone case we may consider, as an example, the function $f(t)=-t^{2}+(1+a) t-a$ with $0<a<\frac{1}{3}$.

\section{References}

[1] A. Ambrosetti \& P.H. Rabinowitz, Dual variational methods in critical point theory and applications, J. Funct. Anal. 14 (1973)349-381.

[2] D. Arcoya \& M. Calahorrano, Multivalued nonpositone problems, Atti Accad. Naz. Lincei Al. Fis. Mat. Natur. Rend. Lincei (9) Mat. Appl. 1 (1990), N. 2, 117-123. 
[3] J. Bebernes \& A. Lacey, Global existence and finite-time blow-up for a class of nonlocal parabolic problems, Advances in Differential Equations, 2(6) (1997)927-953.

[4] J. Bebernes, C. Li \& P. Talaga, Single point blow-up for nonlocal parabolic problems, Physica D, (134) (1999)48-60.

[5] A. Bose \& G.A. Kriegsman, Large amplitude solutions of spatially nonhomogeneous nonlocal reaction-diffusion equations, Methods Appl. Anal. 7 (2000)295-312.

[6] K.J. Brown \& R. Shivaji, Simple proofs of some results in perturbed bifurcation theory, Proc. Roy. Soc. Edin., 93(A) (1982)71-82

[7] E. Caglioti, P.L. Lions, C. Marchiori \& M. Pulvirenti, A special class of stationary flows for two-dimensional Euler equations: A statistical mechanics description, Comm. Math. Phys., 143 (1992)501-525.

[8] G.F. Carrier, On the non-linear vibration problem of the elastic string, Quart. Appl. Math. 3 (1945)157-165.

[9] A. Castro, C. Maya \& R. Shivaji, Nonlinear eigenvalue problems with semipositone structure, Nonlinear Differential Equations, Electron., J. Dif. Eqns., Conf. 05, 2000, pp. 33-49.

[10] A. Castro \& R. Shivaji, Non-negative solutions for a class of nonpositone problems, Proceedings of the Royal Society of Edinburgh, 108A, (1988)291-302.

[11] N. Chafee, The Electric Ballast Resistor: Homogeneous and Nonhomogeneous Equilibria, in Nonlinear Differential Equations: Invariance, Stability and Bifurcation (P. de Mottoni and L. Salvadori, eds.)97-127, Academic Press, New York, 1981. 
[12] K.C. Chang, Variational methods for non-differentiable functionals and their applications to partial differential equations, J. Math. Anal. Appl. 80 (1981) 109-129.

[13] M. Chipot, The diffusion of a population partly driven by its preferences, A.R.M.A. 155 (2000)237-259.

[14] M. Chipot, Remarks on some class of nonlocal elliptic problems, Recent Advances on Elliptic and Parabolic Issues, World Scientific (2006)79102.

[15] M. Chipot, Elements of Nonlinear Analysis, Birkhäuser Advanced Texts 2000 .

[16] M. Chipot \& B. Lovat, Some remarks on nonlocal elliptic and parabolic problems, Nonlinear Anal., 30(7) (1997)4619-4627.

[17] M. Chipot \& B. Lovat, On the asymptotic behaviour of some nonlocal problems, Positivity (1999)65-81.

[18] M. Chipot \& J.F. Rodrigues, On a class of nonloal nonlinear problems, Math. Model. Numer. Anal., 26(3) (1992)447-468.

[19] D.G. Costa, H. Tehrani \& J. Yang, On a variational approach to existence and multiplicity results for semipositone problems, Electron., J. Dif. Eqns., Vol. 2006 (2006), N. 11, 1-10.

[20] A.C. Fowler, I. Frigaard \& S.D. Howison, Temperature surges in current limiting circuits devices, SIAM J. Appl. Mah., 52(4) (1992)998-1011.

[21] P. Freitas \& M. Grinfeld, Stationary solutions of an equation modelling Ohmic heating, Appl. Math. Lett., 7 (1994)1-6. 
[22] J.M. Gomes \& L. Sanchez, On a variational approach to some non-local boundary value problems, Applicable Analysis, Vol. 84, N. 9, September (2005)909-925.

[23] B. Gidas, W.M. Ni \& L. Nirenberg, Symmetry and related properties via the maximum principle, Comm. Math. Phys. 68 (1979)pp. 209-243.

[24] G. Kirchhoff, Mechanik, Teubner, Leipzig, 1883.

[25] G.A. Klaasen \& E. Mitidieri, Standing wave solutions for a system derived from the FitzHugh-Nagumo equations for nerve conduction, SIAM J. Math. Anal., 17, N. 1 (1986)74-83.

[26] G.A. Kriegsmann, Hot spot formation in microwave heated ceramic fibres, IMA J. Appl. Math., 59 (1997)123-148.

[27] A.A. Lacey, Thermal runaway in a non-local problem modeling Ohmic heating: Part I, Euro. J. Appl. Math., 6 (1995)127-144.

[28] J.L. Lions, Quelques Méthodes de Résolution de Problèmes aux Limites Non Linéaires, Dunod, Paris, 1969.

[29] C.B. Morrey, Multiple integrals in calculus of variations, Springer, Berlin (1966).

[30] W.E. Olmstead, S. Nemat-Nasser \& L. Ni, Shear bands as surfaces of discontinuity, J. Mech. Phy. Solids, 42 (1994)697-709.

[31] C.V. Pao, Blowing-up of solutions for a nonlocal reaction-diffusion problem in combustion theory, J. Math. Anal. Appl., 166 (1992)591-600. 